\title{
The role of structural components in the Mueller-Lyer illusion
}

\author{
SUZANNE GREIST-BOUSQUET and H. R. SCHIFFMAN \\ Rutgers, The State University, Busch Campus, New Brunswick, New Jersey 08903
}

\begin{abstract}
In order to assess the role of the structural components of the Mueller-Lyer illusion, subjects reproduced the central extent of standard Mueller-Lyer figures and configural variations. Illusory magnitude of the underestimated wings-in and overestimated wings-out figures was examined with selective amputations of the oblique wings and central line segment (shaft). Variations were presented at $0,45,90$, and $135 \mathrm{deg}$ from vertical. Orientation had no reliable effect on illusory magnitude. Elimination of the shaft effected a decrease in the apparent extent for all variations, presumably due to the addition of the filled-unfilled space illusory effect to the standard Mueller-Lyer effect. A second study corroborated this finding: The decrease in apparent extent consequent to shaft removal occurred independently of any response factor. Selective wing removal differentially decreased the illusory magnitude of the standard MuellerLyer figures; this was discussed with regard to a dual-illusion hypothesis. Finally, variations that contained no intersecting lines produced a significant illusion in the direction of the standard Mueller-Lyer figures, suggesting the involvement of higher level, nonperipheral distortion mechanisms.
\end{abstract}

The familiar form of the Mueller-Lyer illusion pattern characteristically effects a measurable error in the apparent length of a central line segment when this segment is appropriately flanked by oblique angles (Figures 1A and 1B). Although a number of investigations have examined the contribution of various components (e.g., length of the oblique angles, the angle of oblique attachment, etc.; see Robinson, 1972, for a review of configural manipulations and a historical review of the Mueller-Lyer illusion), there has been little systematic study to determine the critical stimuli within the basic figure for effecting and affecting the magnitude of the illusion. The effect on the magnitude of the illusion has been examined with the removal of the oblique angles or wings (Day \& Dickinson, 1976a; Tausch, 1962; Warren \& Bashford, 1977 ) and with the omission of the central line segment or shaft (Cooper \& Runyon, 1970). However, of interest is a systematic investigation of the effect of combined figural component amputations on illusory magnitude. Although some of these amputated versions have been previously examined (Coren, Girgus, Erlichman, \& Hakstian, 1976; Pollack, 1964; Porac, Coren, Girgus, \& Verde, 1979), it is nonetheless important to assess in a single investigation the illusory effects for a large subset of configural combinations of wing and shaft components; further-

This research was supported by funds from the Research Council of Rutgers, The State University, Grant 07-2109. Requests for offprints should be sent to H. R. Schiffman, Department of Psychology, Busch Campus, Rutgers, The State University, New Brunswick, New Jersey 08903. more, the subset of figures examined needs to include the most minimal configural variation of the MuellerLyer figure to determine the minimal stimuli that effect the illusion. Finally, the effect of the orientation of the figure, a major variable effecting a number of other geometric illusions (e.g., the horizontal-vertical illusion: Schiffman \& Thompson, 1975; the Poggendorff illusion: Weintraub \& Krantz, 1971), has not been systematically investigated for the Mueller-Lyer illusion and its configural variations.

\section{EXPERIMENT 1}

The following examination was undertaken to determine what structural components are necessary to actuate the Mueller-Lyer illusion by selectively amputating various components of the standard figure and, furthermore, to systematically assess the effect of figural orientation on the magnitude of the illusion.

\footnotetext{
Method

Subjects. Twenty undergraduate students served as subjects. All had corrected or uncorrected visual acuity of $20 / 20$.

Stimuli. Series of the standard Mueller-Lyer figures, each made up of a shaft and attached wings, and configural variations (Figure 1) were reproduced on sheets of $21.6 \times 27.9 \mathrm{~cm}$ white paper. Variations that eliminated the wings of the standard figures (Figures $1 \mathrm{C}$ through $1 F$ ) were selected based on the availability in the literature of data on analogous versions (Warren \& Bashford, 1977).

The linear components of the stimulus figures were constructed from .79-mm-wide black graph tape. The lengths of the shafts of the stimulus figures were constant at $50 \mathrm{~mm}$. The wings of the figures were $15 \mathrm{~mm}$ in length and formed a $45 \mathrm{deg}$ angle with the
} 


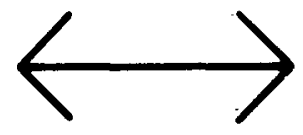

(A)

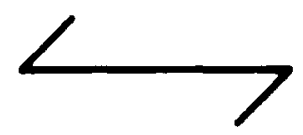

(c)

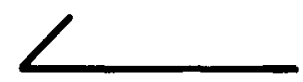

(E)
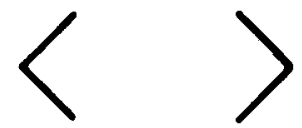

(G)

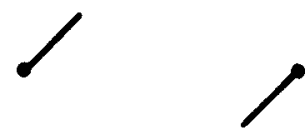

(1)

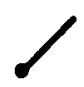

( $k)$

Figure 1. The standard wings-in (A) and wings-ont (B) versions of the Mueller-Lyer illusion and configural variations (C through $L$ ).

shaft in the wings-in conditions and a 135-deg angle with the shaft in the wings-out conditions. The configural variations were similarly constructed, with corresponding amputations of the appropriate components. For variations that omitted the shaft and contained less than the standard four wings (Figures 1I through 1L), black dots $2 \mathrm{~mm}$ in diameter marked the $50-\mathrm{mm}$ linear extents. Two control figures, one that contained only the shaft and one that contained only two boundary dots, were similarly constructed. Thus, there were 14 independent experimental figures in total. The stimulus figure appeared in the center of the left half of the stimulus sheet. An 80 -mm-long response line was centered in the right half of the stimulus sheet for those stimulus figures that included the shaft; a black response dot $1.5 \mathrm{~mm}$ in diameter marked the beginning of the equivalent response extent for stimulus figures which omitted the shaft.

Identical sets of Mueller-Lyer variations and control figures were constructed at orientations of $0,45,90$, and $135 \mathrm{deg}$ from vertical. The response line for each figure was oriented in correspondence with the stimulus figure, and the response dot was located at the top or leftmost point in correspondence with the linear extent to be judged.

Fourteen nonexperimental control figures, identical to the stimulus and control figures but with different shaft lengths, were also used. The length was increased by $10 \mathrm{~mm}$ in seven of the control figures and decreased by $10 \mathrm{~mm}$ in the remaining seven figures. The four orientations were approximately equally represented. The assignments were random and independent.

Procedure. The stimulus series was presented in a folder to the subject. The subject was instructed to pull the top sheet from the folder, positioning the long side of the stimulus sheet parallel to the desk edge. The shaft extent for the experimental figures subtended approximately $6 \mathrm{deg} 20 \mathrm{~min}$ of visual angle as measured from an average viewing distance of $45 \mathrm{~cm}$. Room illumination was constant at $5.84 \mathrm{~cd} / \mathrm{m}^{2}$. For figures that included the shaft, subjects were instructed to indicate their estimate of the shaft length by placing a slash through the response line such that the length marked matched the linear extent represented in the stimulus figure. The top or leftmost point of the response line was indicated as the starting point. For figures in which the shaft was omitted, the subject was instructed to pencil a small dot relative to the printed response dot on the stimulus sheet to match the length and orientation of the stimulus figure-that is, to place a dot in relation to the given response dot such that the length between the two equaled the length between the vertices of the angles or the dots of the stimulus figure. The printed response dot indicated the top or leftmost starting point. (The subject was allowed to reposition his or her response and was instructed to circle the final response.) After making a response, the subject turned the sheet face down and proceeded with the next sheet from the folder. The subject viewed each figure and made a response within approximately $10 \mathrm{sec}$. Measurements, drawing lines, and movements of the stimulus sheet were not permitted.

Separate presentation sequences for each subject were randomly determined for the 56 experimental stimuli. The 14 control figures in which the shaft length was varied were randomly interleaved between the experimental stimuli for a total of 70 judgments. These were included solely to reduce the possibility of the subject's concluding that all the lengths presented were equal. The randomized presentation of figures was designed to distribute previously found, but limited, decrement effects (ranging from $25 \%$ to $40 \%$; see Coren \& Girgus, 1972b, 1972c; Girgus, Coren, Durant, \& Porac, 1975; Girgus, Coren, \& Horowitz, 1973) across the variations examined while permitting a within-subjects design. The design was chosen because it allows each subject to serve as his or her own control, an essential but conservative methodology appropriate to parametric investigations of the Mueller-Lyer illusion (cf. Day \& Dickinson, 1976a; Erlebacher \& Sekuler, 1969; Pressey \& Di Lollo, 1978; Restle \& Decker, 1977; Sekuler \& Erlebacher, 1971).

\section{Results}

The illusory distortion induced by each of the figures was assessed by measuring the linear extent produced by the subject (to the nearest millimeter). Separate analyses of variance were performed on the length judgments for the wings-in and wings-out 
Mueller-Lyer figures. The results are presented for each of the configural manipulations.

Orientation. Orientation did not have a reliable effect on the distortion induced by the wings-in $[F(3,57)$ $=1.71, p>.174]$ and wings-out $[F(3,57)=1.13$, $\mathrm{p}>.3459$ ] versions of the Mueller-Lyer figure.

Shaft. The presence of the shaft was found to have a significant effect on the magnitude of the illusion for both Mueller-Lyer versions. Specifically, when the shaft was removed in the wings-out figure, a significant reduction in overestimation was found to occur $[F(1,19)=13.00, p<.0019]$. For the wings-in figure, a significant increase in underestimation was observed with the removal of the shaft $[F(1,19)=$ $19.99, \mathrm{p}<.0003$ ]. That is to say, the absence of the shaft effected a decrease in apparent length for both wings-in and wings-out versions of the Mueller-Lyer illusion.

The interaction of shaft by orientation was found to be significant for the wings-out Mueller-Lyer configuration $[F(3,57)=5.53, p<.0022]$; this was not observed for the wings-in figure $[F(3,57)=1.89$, $p>.1404]$. The significant interaction may be due, in large part, to a robust difference in distortion that was noted with the removal of the shaft in the 135-deg orientation; this large difference was also observed for the wings-in configuration and for the control figure differences.

Wing amputation. The main effect of wing amputation was found to be significant for both the wingsin $[F(3,57)=21.31, p<.0001]$ and wings-out $[F(3,57)$ $=62.14, \mathrm{p}<.0001]$ Mueller-Lyer variants. Further analyses used Bonferroni (Kirk, 1968, p. 79) multiple comparison procedures, and all differences reported were significant at the .05 level.

For the wings-out figure, a significant reduction in overestimation was found when the four-wing figure (see Figures $1 \mathrm{~B}$ and $1 \mathrm{H}$ ) was reduced to the twowing figure (see Figures 1D and 1J). A further significant decrease in illusory magnitude was observed when the two-wing figure was amputated to the onewing figure (see Figures $1 F$ and $1 \mathrm{~L}$ ). Despite the general decrement in overestimation with wing amputation, the single-wing figure was found to induce a significant overestimation in comparison with the control containing no wings. This general trend was observed irrespective of the presence of the shaft in the wingsout figure (see Figure 2), and, indeed, the interaction and other higher order interactions were not found to be significant.

Figure 3 graphs the trends of the underestimation induced by the wings-in figures as a function of number of wings present and the presence or absence of the shaft. Separate comparisons were made on the shaft-absent and shaft-present figures since the interaction was found to be significant $[F(3,57)=2.75$, $\mathrm{p}<.05]$. No other higher order interactions were found to be significant.

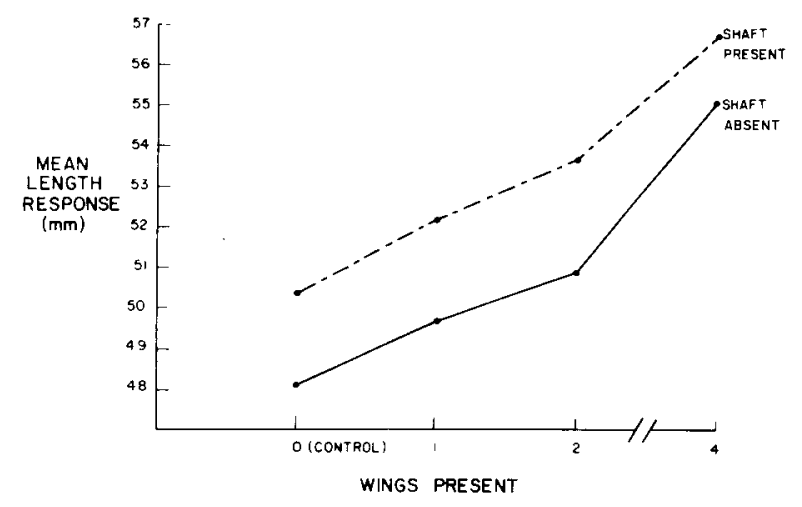

Figure 2. Mean length responses for the wings-out figure as a function of number of wings present and the presence of the shaft.

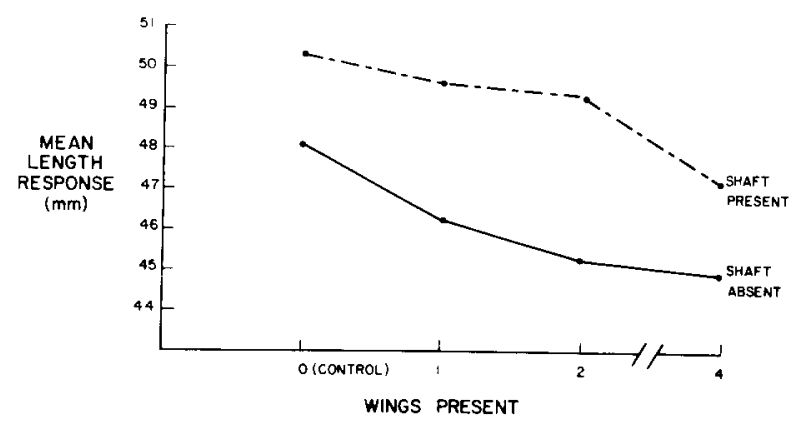

Figure 3. Mean length responses for the wings-in figure as a function of number of wings present and the presence of the shaft.

For wings-in figures that included the shaft, the reduction of the four-wing figure (Figure 1A) to the two-wing figure (Figure 1C) resulted in a significant decrease in underestimation. No significant differences in judged length were observed when the two-wing figure was reduced to the single-wing figure (Figure 1E) and, similarly, when the one-wing figure was compared with the control containing no wings. An additional comparison indicated that the twowing figure did not induce a significant underestimation effect-that is, it was found not to be different from the control.

For wings-in figures that omitted the shaft, the reduction of the four-wing figure (Figure 1G) to the two-wing figure (Figure 1I) resulted in no change in distortion; similarly, the reduction of the two-wing figure to the single-wing figure (Figure 1K) did not result in a significant decrease in underestimation. The single-wing figure was found to induce a significant underestimation effect when compared with the control.

\section{Discussion}

The main findings are: (1) Orientation of the Mueller-Lyer figures has no reliable effect on the 
magnitude of the distortion; (2) removing the central shaft effects a decrease in the apparent extent of wings-in and wings-out Mueller-Lyer figures: that is, there is an increase in the usual underestimation for the wings-in figure and a decrease in the usual overestimation for the wings-out figure; and (3) amputating the wings of the figure was found, in general, to induce a differential decrease in the magnitude of apparent distortion for the two types of figures. Specifically, for the wings-out figure, the systematic reduction in overestimation that was noted with wing amputation occurred equivalently in figures that included and omitted the shaft, whereas for the wingsin figure, the decrease in underestimation was found to differ for figures that included and omitted the shaft. A reduction to zero illusion was noted with the removal of any wings in the shaft-present wings-in figures; however, for the shaft-absent figure, a significant decrease in distortion occurred only when all wings were removed. Most striking is the observation that a single freestanding oblique line (Figures 1K and $1 \mathrm{~L}$ ) was found to induce a significant distortion of apparent extent that is in correspondence with the underestimation and overestimation typically observed with the standard Mueller-Lyer figures. It is of interest to note that, using a somewhat different methodology, Day (1974) reported that an oblique line was found to effect an illusion of linear extent consistent with the latter findings.

Some recent speculation and study concerning the contributing causes of visual illusions are that different processes, in combination, promote systematic perceptual error. One approach has attempted to separate some familiar illusory figures into several elementary illusory effects. For example, Day and Dickinson (1976b) have suggested that one of the contributing factors in the Poggendorff illusion is the horizontal-vertical effect (i.e., the apparent elongation of vertical as opposed to horizontal extent). Another approach has attempted to assess the mode and sequence of the interaction of illusion producing processes by combining illusion figures (Coren \& Ward, 1979).

With regard to combinatorial analyses, Coren and Ward (1979) reported that some illusory effects combine in an additive fashion, while other effects average to produce composite illusory effects. Of particular interest in the context of the present investigation are Coren and Ward's results with regard to the Mueller-Lyer and horizontal-vertical illusions: An additivity of overestimation effects was found. These results confirmed previous findings of $\mathrm{Ni}$ (1934). The disagreement of the present findings (of an absence of any change in illusory magnitude as a result of orientations changes) and previous findings may be due to the different methodologies used. Ni (1934) obtained estimates of the PSE using a method of con- stant stimulus differences, whereas Coren and Ward (1979) employed the method of graded series: Observers chose among a graded series of comparison stimuli to match the stimulus extent. In contrast, the present investigation used the method of reproduction. Although previous comparisons of data yielded by various methodologies have shown that the method of reproduction and the method of graded series adequately measure changes in illusory effects (Coren \& Girgus, 1972a), the appearance of an orientation effect on the magnitude of the Mueller-Lyer illusion may be methodologically dependent.

With regard to reduction analyses of illusory effects, the results of the present investigation suggest the contribution of a filled vs. unfilled space illusion operant with the configural manipulations of the presence vs. absence of the shaft. The filled-unfilled space illusion refers generally to the phenomenon that a filled extent appears longer than an unfilled extent of equal length (Robinson, 1972, p. 49). Previous investigations have implicated this illusory effect in the Mueller-Lyer illusion in that changes in illusory magnitude occur with the addition of multiple wings (Pressey \& Wilson, 1977). However, the filled-unfilled space illusory effect may be more fundamentally involved in the Mueller-Lyer illusion. Indeed, a form of the filled-unfilled space illusion occurs with minimal stimulus requirements: A continuous line appears longer in length than an uninterrupted empty extent (Pressey \& Moro, 1971). The findings described herein are consistent with this notion: The extent of a continuous line (shaft-present condition) was judged consistently greater than the length of an identical extent bounded by oblique lines or dots (shaft-absent condition). This difference in apparent extent was found to occur in all Mueller-Lyer variations examined. That is, in all conditions a decrease in the apparent extent was observed with shaft removal.

\section{EXPERIMENT 2}

The role of response mode in the significant decrement in apparent extent consequent to shaft removal was examined further. Specifically, it was necessary to ensure that the decrease in apparent length found to occur with shaft removal was not due to the response measure used since shaft-absent and standard variations required different responses. That is, shaftabsent variations required a production response in unfilled space (i.e., positioning a dot in relation to a response dot in an otherwise empty field), whereas shaft-present variations required a response in filled space (i.e., placing a slash on a response line). Accordingly, this study was undertaken to examine the influence of the response requirements used in Experiment 1: The production requirements of both 
line and dot responses were used in conjunction with standard and shaftless variations of the Mueller-Lyer.

\section{Method}

Subjects. Twenty new undergraduate students served as subjects. All had corrected or uncorrected visual acuity of $20 / 20$.

Stimuli. Standard and shaftless four-wing Mueller-Lyer figures and line and dot control figures were constructed in accordance with the stimulus dimensions described in Experiment 1. All stimulus figures were horizontally oriented. Each stimulus figure appeared twice in the series, once with the response line and once with the response dot as previously described.

Three nonexperimental control figures, identical to the stimulus figures but with different shaft length, were also used. The length was decreased by $10 \mathrm{~mm}$ in two of these control figures and increased by $10 \mathrm{~mm}$ in the remaining control figure. Each stimulus figure type (i.e., wings-in, wings-out, and control) was represented. The assignments were random and independent.

Procedure. The procedure was identical to that used in Experiment 1 . Subjects were instructed to indicate their estimate of the stimulus length as the response procedure required. That is, when a response line appeared, subjects were instructed to place a slash through the line to indicate their length estimates; likewise, when a response dot appeared, subjects were instructed to laterally position a small dot to match the stimulus length.

Separate presentation sequences for each subject were randomly determined for the 12 experimental stimuli. The three nonexperimental control figures in which the shaft length was varied were randomly interleaved between the experimental stimuli for a total of 15 judgments.

\section{Results}

The illusory distortion induced by each stimulus figure was assessed by measuring the linear extent produced by the subject (to the nearest millimeter). A single analysis of variance was performed on the length judgments.

The main effect of figure type was found to be significant $[F(2,38)=46.48, p<.0001]$. A Bonferrori multiple comparison procedure $(\alpha=.05)$ revealed that the wings-in Mueller-Lyer figure effected a significant underestimation of linear extent in comparison with control figure judgments; likewise, wingsout Mueller-Lyer figures were significantly overestimated in comparison with control figure judgments. Figure 4 illustrates these findings.

The presence of the shaft was found to have a significant effect on illusory magnitude $[F(1,19)=10.68$, $p<.004]$. As graphically depicted in Figure 4, wingsin, wings-out, and control figure judgments were subject to a decrement in apparent extent consequent to shaft removal. However, the specific response requirement (response production in minimally filled or unfilled space) did not significantly influence illusory magnitude $[F(1,19)=11.267, p>.66]$. No interaction of the main effects was significant.

\section{Discussion}

Based upon the findings of Experiment 2, it is clear that shaft removal effects a significant reduction of apparent extent independent of the response requirement. The minimal form of the filled-unfilled

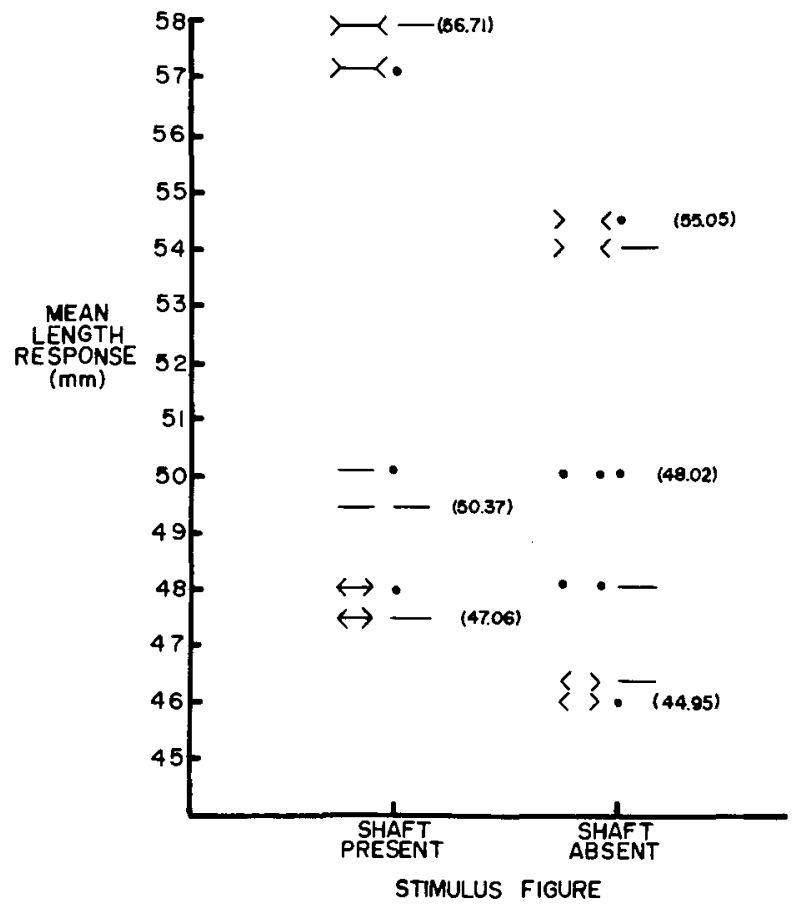

Figure 4. Mean length responses for the wings-in, wings-out, and control figures as a function of the presence of the shaft and response procedure required. The data points are represented symbolically by the actual stimulus figure and response mode use (i.e., line vs. dot). Values in parentheses refer to the corresponding findings from Experiment 1 for comparison.

space illusion was found: An uninterrupted empty extent (i.e., the control figure containing boundary dots) was generally judged shorter than an equivalent continuous line (the control line). This is consistent with the findings of Pressey and Moro (1971). Moreover, this minimal form of the filled-unfilled space illusion was found to influence the illusory magnitude of standard forms of the Mueller-Lyer illusion: When the shaft of the Mueller-Lyer figure is eliminated, a significant decrease in apparent extent occurs. Thus, removal of the shaft enhances the underestimation of the wings-in Mueller-Lyer illusion and detracts from the overestimation of the wings-out illusion. The response requirement neither interfered with nor caused this basic effect.

\section{GENERAL DISCUSSION}

The increase in underestimation and decrease in overestimation found here to occur with the removal of the shaft in the wings-in and wings-out MuellerLyer figures, respectively, has been previously reported by other investigators (Cooper \& Runyon, 1970). This finding has been interpreted as support for the suggestion made by Binet as early as 1895 (see Robinson, 1972) that the Mueller-Lyer illusion 
consists of two discrete illusions-a wings-in and wings-out illusion (Sekuler \& Erlebacher, 1971). Although the two Muellor-Lyer figures appear to produce opposite changes in illusory magnitude with shaft removal, it is here suggested that the effects are due rather to the addition of the filled-unfilled space illusion to the traditional Mueller-Lyer effect.

Support for a dual-illusion hypothesis is suggested by the differential changes in illusory magnitude that were observed for the Mueller-Lyer wings-in and wings-out figures with systematic wing amputation in the first study and in previous studies (e.g., Day \& Dickinson, 1976a; Warren \& Bashford, 1977). A systematic reduction in apparent expansion was found with the successive removal of the wings in the wingsout figure: The decreases in the apparent extent of the shaft-present wings-out figure were $3.04,4.54$, and $6.34 \mathrm{~mm}$ when two wings, three wings, and four wings, respectively, were amputated. In contrast, for the wings-in figure, previous investigators have found no decrease in underestimation with removal of two wings (Warren \& Bashford, 1977) or a decrease that is dependent upon the placement of the remaining wings (Day \& Dickinson, 1976a). Furthermore, a decrease in illusory magnitude has been reported for the removal of three wings, yet the degraded figure was still significantly underestimated (Day \& Dickinson, 1976a; Warren \& Bashford, 1977). The present results, using similar stimulus figures (the wings-in figures with a shaft), are not in complete agreement (i.e., the underestimation was eliminated with the removal of two and three wings); this may be due to the different configural parameters and procedures employed herein. However, the findings reported here indicate the importance of the placement of the wings in determining the magnitude of the wings-in Mueller-Lyer illusion.

Indeed, an explanation proposed for the wings-in version of the illusion is that it is due to a perceptual confusion of the span between the wing tips with the distance to be judged (Erlebacher \& Sekuler, 1969; Sekuler \& Erlebacher, 1971). Specifically, for the standard wings-in figure, the distance between the tips of wings has been found to be a significant determinant of the illusory underestimation suggesting that subjects incorporate this distance into their judgments of the extent between the vertices of the angles. However, for the standard wings-out figure, the magnitude of the illusion has been found to be unaffected by intertip distance. This latter finding indicates that a confusion theory does not provide an adequate account for the effect of the wings-out Mueller-Lyer version, thereby arguing for a dualillusion notion for the presumed unitary Mueller-Lyer illusion (Sekuler \& Erlebacher, 1971).

In amputating the wings of the Mueller-Lyer figures, the present study eliminated the effect of inter- tip distance (see Figures 1C through 1F). Indeed, for the wings-in versions that included a shaft, the illusion was effectively abolished with the removal of any of the wings. In contrast, although decreases in overestimation were found to occur with the systematic amputation of wings in the wings-out version, a reliable illusory effect remained. These findings suggest that confusion of the intertip distance may play a role only in the distortion induced by the wings-in Mueller-Lyer figure.

Some of the findings of the present study suggest the contribution of a higher level distortion mechanism in the Mueller-Lyer illusion. A recent approach to explaining the processes involved in the visual illusions is that they result from a combination of distortion mechanisms that function at various physical and conceptual loci or levels within the visual system (Coren \& Girgus, 1978a, 1978b; Coren, Porac, \& Ward, 1979; Coren \& Ward, 1979). Thus, at the more peripheral levels, optical and retinal factors are presumed operative with illusion figures that contain intersecting lines (Chiang, 1968; Coren, 1969, 1970; Coren \& Ward, 1978; Robinson, 1968; Ward \& Coren, 1976). According to this notion, degraded versions of the Mueller-Lyer illusion that contain no intersecting lines (e.g., Figures $1 \mathrm{I}, 1 \mathrm{~K}, 1 \mathrm{~J}, 1 \mathrm{~L}$ ) bypass the optical and retinal loci of distortion, yet all were here found to reflect a significant illusory distortion in correspondence with that typically observed with the standard Mueller-Lyer figures. This can be interpreted as implicating contributing sources of distortion beyond the peripheral levels for both wings-in and wings-out Mueller-Lyer figures.

However, for the wings-out figure, the significant reduction in illusory magnitude with the elimination of intersecting lines (Figures $1 \mathrm{~J}$ and $1 \mathrm{~L}$ ) is consistent with the notion that the proposed peripheral factors may contribute to the standard illusory overestimation effect. No such reduction in distortion occurred when intersecting lines were removed in the wings-in figure (Figures $1 \mathrm{I}$ and $1 \mathrm{~K}$ ). These results, then, further support the notion that the Mueller-Lyer illusion may involve two discrete illusions and that the apparent expansion of the wings-out configuration may be due to an interplay of distortion effects that are actuated at different loci within the visual system. Accordingly, it is here suggested that future examination of the optical and retinal sources of distortion in the MuellerLyer illusion treat the standard figure as being made up of two independent figures-that is, one should study separate versions of the figure (wings-in and wings-out) rather than the Brentano (or combined) figure that has been previously used (Coren, 1970).

In summary, the present findings indicate that the Mueller-Lyer illusion is not a simple line configuration that is explicable by a unitary mechanism. Rather, the illusory configuration may be promoted by the 
unfilled-filled space illusion in addition to the MuellerLyer effect. Furthermore, the apparently similar Mueller-Lyer wings-in and wings-out versions may consist of distinct illusions and may differentially involve contributing sources of distortion within the visual system.

\section{REFERENCES}

Chinang, C. A new theory to explain geometric illusions produced by crossing lines. Perception \& Psychophysics, 1968, 3, 174-176.

Cooper, M. R., \& Runyon, R. P. Error increase and decrease in minimal form of Mueller-Lyer illusion. Perceptual and Motor Skills, 1970, 31, 535-538.

Coren, S. The influence of optical aberrations on the magnitude of the Poggendorff illusion. Perception \& Psychophysics, 1969, 6, 185-186.

COREN, S. Lateral inhibition and geometric illusions. Quarterly Journal of Experimental Psychology, 1970, 22, 274-278.

Conen, S., \& Girgus, J. S. A comparison of five methods of illusion measurement. Behavior Research Methods \& Instrumentation, 1972, 4, 240-244. (a)

Coren, S., \& Girqus, J. S. Differentiation and decrement in the Mueller-Lyer illusion. Perception \& Psychophysics, 1972, 12, 466-470. (b)

Coren, S., \& Girgus, J. S. Illusion decrement in intersecting line figures. Psychonomic Science, 1972, 26, 108-110. (c)

Coren, S., \& Girgus, J. S. Seeing is deceiving: The psychology of visual illusion. Hillsdale, N.J: Erlbaum, 1978. (a)

Coren, S., \& Girgus, J. S. Visual illusions. In R. Held, H. Leibowitz, \& H. L. Teuber (Eds.), Handbook of sensory psychology (Vol. 8): Perception. Berlin: Springer, 1978. (b)

Coren, S., Girgus, J. S., Erlichman, H., \& Hakstian, A. R. An empirical taxonomy of visual illusions. Perception \& Psychophysics, 1976, 20, 129-137.

Coren, S., Porac, C., \& Ward, L. M. Sensation and perception. New York: Academic Press, 1979.

Coren, S., \& WARD, L. M. The effect of optical blur on visualgeometric illusions. Bulletin of the Psychonomic Society, 1978, 11, 390-392.

Coren, S., \& WARD, L. M. Levels òf processing in visual illusions: The combination and interaction of distortion producing mechanisms. Journal of Experimental Psychology: Human Perception and Performance, 1979, 5, 324-335.

DAY, R. H. The Poggendorff illusion: Apparent displacement of the oblique lines. Australian Journal of Psychology, 1974, 26, 49-59.

DAY, R. H., \& Dickinson, R. G. Apparent length of the arms of acute and obtuse angles, and the components of the Müller-Lyer illusion. Australian Journal of Psychology, 1976, 28, 137-148. (a)

DAY, R. H., \& Dickinson, R. G. The components of the Poggendorff illusion. British Journal of Psychology, 1976, 67, 537-552. (b)

Erlebacher, A., \& Sekuler, R. Explanation of the Muellet-
Lyer illusion: Confusion theory examined. Journal of Experimental Psychology, 1969, 80, 462-467.

Girgus, J. S., Conen, S., Durant, M., \& Porac, C: The assessment of components involved in illusion formation using a longterm decrement procedure. Perception \& Psychophysics, 1975, 18, 144-148.

Girovs, J. S., Conen, S., \& Honowitz, L. Peripheral and central components in variants of the Mueller-Lyer illusion. Perception \& Psychophysics, 1973, 13, 157-160.

Kırk, R. E. Experimental design: Procedures for the behavioral sciences. Belmont, Calif: Wadsworth, 1968.

Ni, C. F. The effect of combining some geometric optical illusions. Journal of General Psychology, 1934, 10, 472-476.

Pollack, R. H. The effects of fixation in the apparent magnitude of bounded horizontal extents. American Journal of Psychology, 1964, 77, 177-192.

Porac, C., Coren, S., Girgus, J. S., \& Verde, M. Visualgeometric illusions: Unisex phenomena. Perception, 1979, 8 , $401-402$.

Pressey, A. W., \& Di Lollo, V. Effects of distance between standard and comparison lines on the Mueller-Lyer illusion. Perception \& Psychophysics, 1978, 24, 415-419.

Pressey, A. W., \& Moro, T. L. An explanation of Cooper and Runyon's results on the Mueller-Lyer illusion. Perceptual and Motor Skills, 1971, 32, 564-566.

Pressey, A. W., \& Wilson, A. E. The hydra-headed MuellerLyer effect: A theoretical puzzle. Perception \& Psychophysics, 1977, 22, 392-394.

Restle, F., \& Decker, J. Size of the Mueller-Lyer illusion as a function of its dimensions: Theory and data. Perception \& Psychophysics, 1977, 21, 489-503.

Roвinson, J. O. Retinal inhibition in visual distortion. British Journal of Psychology, 1968, 59, 29-36.

RoBinson, J. O. The psychology of visual illusion. London: Hutchinson, 1972.

Schiffman, H. R., \& Thompson, J. G. The role of figure orientation and apparent depth in the perception of the horizontal vertical illusion. Perception, 1975, 4, 79-83.

Sekuler, R., \& Erlebacher, A. The two illusions of MüllerLyer: Confusion theory reexamined. American Journal of Psychology, 1971, 84, 477-486.

TAusch, R. Empirical investigations regarding perceptual interpretations in the field of holistic and Gestalt psychology. Zeitschrift für Psychologie, 1962, 166, 26-61 (in German).

WARD, L. M., \& Coren, S. The effect of optically induced blur on the magnitude of the Mueller-Lyer illusion. Bulletin of the Psychonomic Society, 1976, 7, 483-484.

Warren, R. M., \& Bashrord, J. A. Müller-Lyer illusions: Their origin in processes facilitating object recognition. Perception, 1977, 6, 615-626.

Weintraub, D. J., \& Krantz, D. H. The Poggendorff illusion: Amputations, rotations, and other perturbations. Perception \& Psychophysics, 1971, 10, 257-263.

(Manuscript received February 17, 1981; revision accepted for publication July 30,1981 .) 